\title{
HOWE/KIRILLOV THEORY FOR $p$-ADIC SYMMETRIC SPACES
}

\author{
JEFFREY HAKIM \\ (Communicated by Dennis A. Hejhal)
}

\begin{abstract}
The method of coadjoint orbits is adapted to sufficiently small compact subgroups of a pair $\left(G, G_{+}\right)$, where $G$ is a $p$-adic group and $G_{+}$is the subgroup of fixed points of an involution. The techniques developed here have been used to prove local integrability of certain distributions which are fundamental in the harmonic analysis of $G / G_{+}$.
\end{abstract}

\section{INTRODUCTION}

Howe's $p$-adic generalization of the Kirillov "orbit method" [5] gives a correspondence between representations of a sufficiently small compact, open subgroup $K$ of a group $\mathbf{G}(F)$ (the $F$-points of a linear algebraic group $\mathbf{G}$ defined over a $p$-adic field $F$ ) and coadjoint orbits in the dual of the Lie algebra $\mathfrak{g}$ of $\mathbf{G}(F)$. The correspondence is expressed in terms of a character formula. In this paper, we present a related correspondence and character formula for $p$-adic symmetric spaces.

Assume $\sigma$ is an $F$-linear automorphism of order two on $\mathfrak{g}$ and $L$ is a $\sigma$ stable e.e. lattice in $\mathfrak{g}$. (The definition of "e.e. lattice" is recalled in the next section.) If $K$ is the group $\exp L$, then $\sigma$ defines via the exponential map an involution of $K$. Let $K_{+}$denote the subgroup of $\sigma$-fixed points in $K$. We show in Theorem 1 that $\left(K, K_{+}\right)$is a Gelfand pair. Hence, the dimension of the space of $K_{+}$-fixed vectors of any irreducible representation of $K$ is at most one. When the dimension is one, we say the representation is "spherical". In Theorem 3, a correspondence between the spherical representations of $K$ and certain coadjoint orbits is established. The associated character formula involves the spherical function of $d$ with respect to $\sigma$.

An interesting special case arises as follows. Let $E$ be a quadratic extension of $F$, and let $\mathbf{H}$ be a linear algebraic $F$-group. Take $\mathbf{G}$ to be the $F$-group obtained by first regarding $\mathbf{H}$ as an $E$-group and then applying Weil's restriction of scalars from $E$ to $F$. Thus $\mathbf{G}(F)=\mathbf{H}(E)$, and $\sigma$ is given by applying Galois conjugation for $E / F$ to the coordinates of $\mathbf{H}(E)$. The correspondence of Theorem 3 yields a correspondence between the irreducible representations $d_{+}$of $K_{+}$and the spherical representations $d$ of $K$. The character of $d_{+}$is

Received by the editors August 20, 1992 and, in revised form, November 25, 1992.

1991 Mathematics Subject Classification. Primary 22E35, 22E55, 43 A95.

Partially supported by NSA Grant MDA904-92-H-3056. 
related to the spherical function of $d$, and the dimension of $d$ is the square of the dimension of $d_{+}$. (See Theorem 4.)

These results are applied to the theory of automorphic representations in [2]. In particular, in generalizations of the Jacquet-Lai relative trace formula [3, 6], one encounters a distribution analogous to the character of a local admissible representation. In much the same way that Harish-Chandra applied Howe's results to prove local integrability of characters of admissible representations, our orbit method can be used to prove local integrability of these "relative characters". This is done in [2].

The relative character is defined as follows. Assume $\mathbf{H}$ is a connected reductive $F$-group, and let $G=\mathbf{H}(E)$ and $G_{+}=\mathbf{H}(F)$. Suppose $(\pi, V)$ is an irreducible representation of $G$ which admits a nonzero $G_{+}$-invariant linear form $\tilde{\lambda}$. Suppose $(\tilde{\pi}, \tilde{V})$ is the contragredient and $\lambda$ is a nonzero $G_{+}$-invariant form on $\tilde{V}$. Then $\pi(f) \lambda \in V$ when $f \in C_{c}^{\infty}(G)$, and therefore the generalized matrix coefficient

$$
\boldsymbol{\Theta}_{\lambda, \tilde{\lambda}}(f)=\langle\pi(f) \lambda, \tilde{\lambda}\rangle
$$

defines a bi- $G_{+}$-invariant distribution on $G$. Such a distribution is "admissible" in the sense of [4]. In extending the theory of relative trace formula to groups other than $\mathrm{GL}_{2}$, one needs to know that this distribution arises from a locally integrable function.

\section{GelfaND PAIRS}

Throughout this paper, we adopt the notation of the second paragraph of the introduction with the additional requirement that the residue field of $F$ has odd order. Hence, $F$ is a finite extension of some field $\mathbb{Q}_{p}$ of $p$-adic numbers for some odd prime $p$. Let $R$ denote the ring of integers of $F$, and fix a uniformizing element $\varpi \in R$. We assume that the Lie algebra $\mathfrak{g}$ is contained in $\mathfrak{g l}_{n} F$ for some $n$. If $\alpha=\left[\operatorname{ord}_{F}(p) /(p-1)\right]+1$, where [] is the greatest integer function, then the exponential map gives a homeomorphism from $M_{n}\left(\varpi^{\alpha} R\right)$ to $1+M_{n}\left(\varpi^{\alpha} R\right)$. Define another constant $\beta$ to be $\left[3 \operatorname{ord}_{F}(p) / 2(p-1)\right]+1$ if $p \neq 3$ and $\operatorname{ord}_{F}(3)+1$ if $p=3$. Following Howe, we say that an $R$-lattice $\mathscr{A} \subset \mathfrak{g} \cap M_{n}\left(\varpi^{\alpha} R\right)$ which is closed under Lie brackets is "e.e." (elementarily exponentiable) if $[\mathscr{A}, \mathscr{A}] \subseteq \varpi^{\beta} \mathscr{A}$. The Campbell-Hausdorff formula then implies that $\exp \mathscr{A}$ defines a group. (See [5].)

With $L$ and $K$ fixed as above, let $L_{+}$and $L_{-}$denote the eigenspaces of 1 and -1 , respectively, in $L$ and put $K_{+}=\exp L_{+}$and $K_{-}=\exp L_{-}$. It is convenient to let $k^{-\sigma}$ denote $\left(k^{-1}\right)^{\sigma}$ for $k \in K$.

Lemma 1. Each element $k \in K$ has a unique expression $k=k_{-} k_{+}$, where $k_{-} \in K_{-}$and $k_{+} \in K_{+}$. Furthermore, $k_{-}=\left(k k^{-\sigma}\right)^{1 / 2}$ and $k_{+} k^{-\sigma} k_{+}=k$.

Proof. If $k \in K$ and $k_{-}=\left(k k^{-\sigma}\right)^{1 / 2}$, then $k_{-} \in K_{-}$. Let $k_{+}=k_{-}^{-1} k$. We have $k_{+}^{\sigma}=\left(k^{\sigma} k^{-1}\right)^{-1 / 2} k^{\sigma}=\left(k k^{-\sigma}\right)^{1 / 2} k^{\sigma}=\left(k k^{-\sigma}\right)^{-1 / 2}\left(k k^{-\sigma}\right) k^{\sigma}=k_{+}$. Hence $k_{+} \in K_{+}$. Now suppose $k=\tilde{k}_{-} \tilde{k}_{+}$is another such decomposition. Then $k k^{-\sigma}=\tilde{k}_{-}^{2}=k_{-}^{2}$. Therefore, $\tilde{k}_{-}=k_{-}$and consequently $\tilde{k}_{+}=k_{+}$. This proves uniqueness. Finally, we note that $k_{+} k^{-\sigma} k_{+}=k_{+}\left(k_{+}^{-1} k_{-}\right) k_{+}=k_{-} k_{+}=k$.

It follows from Lemma 1 that the anti-involution $\theta$ of $K$ defined by $k^{\theta}=$ $k^{-\sigma}$ stabilizes $K_{+}$and acts trivially on $K_{+} \backslash K / K_{+}$. Thus "Gelfand's Lemma" [1, Lemma 2.1] implies 
Theorem 1. If $d$ is an irreducible, spherical representation of $K$, then its space of $K_{+}$-fixed vectors has dimension one.

This theorem is identical to the statement that $\left(K, K_{+}\right)$is a "Gelfand pair". For details and references, see [1].

\section{CHARACTER FORMUlaS}

In order to recall Howe's coadjoint orbit correspondence, we introduce some notation. Whenever $H$ is a compact group, $\widehat{H}$ will denote the collection of irreducible, unitary representations of $H$ up to equivalence, and when $d \in \widehat{H}$, its character is denoted $\xi_{d}$. If $K$ and $L$ are as above, then $K$ acts on $\widehat{L}$ by $\operatorname{Ad}^{*}(k) \chi(X)=\chi\left(\operatorname{Ad}\left(k^{-1}\right) X\right)$.

Theorem 2 (Howe [5]). There is a bijection between representations $d \in \widehat{K}$ and $K$-orbits $\mathscr{O}$ in $\widehat{L}$ such that when $d$ corresponds to $\mathscr{O}$

$$
\xi_{d}(\exp X)=\operatorname{dim}(d)^{-1} \sum_{\chi \in \mathscr{O}} \chi(X)
$$

for all $X \in L$.

We now turn to the analogue of Howe's correspondence for the symmetric space $K / K_{+}$. It is easily seen that if $H^{\prime}$ is a normal subgroup of a compact group $H$ and $d$ is an irreducible representation of $H$, then $d$ possesses a nonzero $H^{\prime}$-fixed vector exactly when $d$ has trivial restriction to $H^{\prime}$. Con-

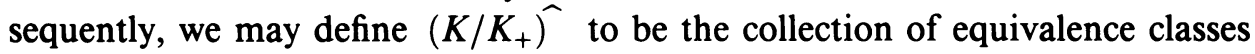
of irreducible spherical representations of $K$ with no risk of confusion when $K / K_{+}$is a group.

Let $\mathfrak{g}_{+}$and $\mathfrak{g}_{-}$denote the eigenspaces of $\sigma$ in $\mathfrak{g}$ for the eigenvalues 1 and -1 , respectively. More generally, if $\mathscr{A}$ is a $\sigma$-stable subalgebra of $\mathfrak{g}$, we use the notation $\mathscr{A}_{+}=\mathscr{A} \cap \mathfrak{g}_{+}$and $\mathscr{A}_{-}=\mathscr{A} \cap \mathfrak{g}_{-}$. If $\mathscr{G}=\exp \mathscr{A}$, we take $\mathscr{G}_{+}=\exp \mathscr{A}_{+}$and $\mathscr{G}_{-}=\exp \mathscr{A}_{-}$, even though these objects are not necessarily groups.

If $d \in \widehat{K}$ then the spherical function $\xi_{d}^{+}$of $d$ is the function on $K$ defined by $\xi_{d}^{+}(k)=\int_{K_{+}} \xi_{d}\left(k k_{+}\right) d k_{+}$, where $d k_{+}$is the Haar measure on $K_{+}$which gives $K_{+}$measure 1. It is easily shown that $\xi_{d}^{+} \not \equiv 0$ if and only if $d \in\left(K / K_{+}\right)$. Furthermore, two representations in $\left(K / K_{+}\right)^{\hat{\gamma}}$ are isomorphic precisely when their spherical functions are identical. It follows from Theorem 1 that if $d$ is unitary and $e_{+}$is a $K_{+}$-fixed unit vector, then the spherical function is identical to the matrix coefficient $\left(d(k) e_{+}, e_{+}\right)$.

The action of $K$ on $\widehat{L}$ restricts to an action of $K_{+}$on $\widehat{L}_{-}$. We will routinely identify characters of $L_{-}$with characters of $L$ which are trivial on $L_{+}$. We prove

Theorem 3. There is a bijection between representations $d \in\left(K / K_{+}\right)$and $K_{+}-$ orbits $\mathscr{O}$ in $\widehat{L}_{-}$such that when $d$ corresponds to $\mathscr{O}$

$$
\xi_{d}^{+}\left(\exp X_{-}\right)=\operatorname{dim}(d)^{-1} \sum_{\chi \in \mathscr{O}} \chi\left(X_{-}\right)
$$

for all $X_{-} \in L_{-}$. 
We remark that evaluating at $X_{-}=0$ in Theorem 3 shows that the order of the orbit $\mathscr{O}$ is equal to the dimension of the spherical representation $d$.

Let us now consider the special case mentioned above which is associated to a quadratic extension $E$ of $F$. Let $R_{E}$ denote the ring of integers of $E$. We may fix an element $l \in R_{E}$ of trace zero which is either a uniformizer or a prime element in $R_{E}$ depending on whether $E / F$ is ramified or not. Then $\{1, l\}$ is an $R$-basis of $R_{E}$. We assume $L_{+}$is an $R$-lattice in $\mathfrak{g}_{+}$and $L=$ $L_{+} \otimes_{R} R_{E}$ is the corresponding $R_{E}$-lattice in $\mathfrak{g}$. We may identify $L_{+}$with $L_{-}$ via $X_{+} \mapsto \imath X_{+}$. Howe's theorem, applied to $K_{+}$and $L_{+}$instead of $K$ and $L$, relates representations of $K_{+}$to characters of $L_{+}$or, equivalently, characters of $L_{-}$. Theorem 3 then relates characters of $L_{-}$to spherical representations of $K$. Combining these correspondences gives

Theorem 4. There is a bijection between representations $d_{+} \in \widehat{K}_{+}$and representations $d \in\left(K / K_{+}\right)$such that when $d_{+}$corresponds to $d$

$$
\operatorname{dim}\left(d_{+}\right)^{-1} \xi_{d_{+}}\left(\exp X_{+}\right)=\xi_{d}^{+}\left(\exp \imath X_{+}\right)
$$

for all $X_{+} \in L_{+}$. Furthermore, the dimension of $d$ is the square of the dimension of $d_{+}$.

Corollary. If $d$ is an irreducible, spherical representation of $K$, then the dimension of $d$ is a square.

We note that the correspondence in Theorem 4 depends on the choice of $l$. This choice is uniquely determined up to multiplication by elements of $R^{\times}$, the group of units in $R$. Via its action on $L$, the group $R^{\times}$acts on $\widehat{K}$ and $\left(K / K_{+}\right)^{-}$. Theorem 4 gives a canonical identification of $R^{\times}$-orbits in $\widehat{K}$ and $\left(K / K_{+}\right)$.

We also mention that $d_{+}$occurs with multiplicity $\operatorname{dim}\left(d_{+}\right)$in the regular representation of $K_{+}$and that $d$ occurs with multiplicity one in the regular representation of $K / K_{+}$. The identity $\operatorname{dim}\left(d_{+}\right)^{2}=\operatorname{dim}(d)$ may be interpreted as a statement of the equality of the dimensions of the isotypic components of $d_{+}$and $d$ in the respective regular representations.

\section{THE ORBIT METHOD}

If $\mathscr{A}$ is an e.e. algebra, then the Campbell-Hausdorff formula implies that the exponential map takes Haar measures on $\mathscr{A}$ to Haar measures on $\exp \mathscr{A}$. Therefore, in view of Theorem 1, if one averages Howe's character formula over $L_{+}$with respect to a Haar measure, one obtains

Lemma 2. Assume $d \in \widehat{K}$, and let $\mathscr{O}_{d}$ be the corresponding $K$-orbit in $\widehat{L}$. If $d$ is spherical, then

$$
\operatorname{dim}(d)=\#\left(\mathscr{O}_{d} \cap \widehat{L}_{-}\right)
$$

Otherwise, $\mathscr{\sigma}_{d}$ and $\widehat{L}_{-}$are disjoint.

Fix $\chi \in \widehat{L}_{-}$. Let $I$ denote the lattice of all $X \in L$ such that $\chi([X, Y])=1$ for all $Y \in L$. As in [5], we say a lattice $J$ "polarizes" $\chi$ if $I \subset J \subset L$, $\#(L / J)=\#(J / I)$, and $\operatorname{Ad}^{*}(\exp X) \chi(Y)=\chi(Y)$ for all $X, Y \in J$. 
Lemma 3. The algebra $J=I+L_{+}$polarizes $\chi$.

Proof. Arguing as in the proof of Lemma 1.1 in [5], one shows that $\operatorname{Ad}^{*}(\exp X) \chi(Y)=\chi(Y)$ for all $X, Y \in J$ if and only if $\chi([X, Y])=1$ for all $X, Y \in J$. The latter condition is clear.

Showing that $\#(L / J)=\#(J / I)$ is equivalent to showing that $\#\left(L_{-} / I_{-}\right)=$ $\#\left(L_{+} / I_{+}\right)$. Let $t$ be the smallest nonnegative integer such that $\varpi^{t} L \subset I$. If $r$ is any integer with $0 \leq r \leq t$, then let $V_{r}=I+\varpi^{r} L$ and $V_{r}^{\perp}=\varpi^{-r} I \cap L$. One may easily check for $1 \leq r \leq t$ that if $X \in V_{r-1}$ and $Y \in V_{r}^{\perp}$, then $\chi([X, Y])$ defines a nondegenerate pairing between $V_{r-1} / V_{r}$ and $V_{r}^{\perp} / V_{r-1}^{\perp}$. Both of these quotient spaces are finite-dimensional vector spaces over the residue field of $F$. Since the pairing is nondegenerate, the dimensions of these vectors spaces are the same.

Let $W_{r}^{+}$denote the fixed points of $\sigma$ in $V_{r-1} / V_{r}$, and let $W_{r}^{-}$denote the -1 eigenspace for $\sigma$ in $V_{r}^{\perp} / V_{r-1}^{\perp}$. Since there is a nondegenerate pairing between $W_{r}^{+}$and $W_{r}^{-}$, these spaces have the same dimension over the residue field. It now follows from the decompositions $L_{+} / I_{+} \cong \bigoplus W_{r}^{+}$and $L_{-} / I_{-} \cong \bigoplus W_{r}^{-}$ that $\#\left(L_{-} / I_{-}\right)=\#\left(L_{+} / I_{+}\right)$.

Regarding $\chi$ as a character of $K$, we see from Theorem 2 that there is a unique representation $d \in \widehat{K}$ such that $\chi$ belongs to the associated $K$-orbit $\mathscr{O}_{d}$ in $\widehat{L}$. The representation $d$ is constructed in [5] as follows. Let $B=$ $\exp J$. Then $\eta(\exp X)=\chi(X)$ defines a character $\eta$ of the group $B$. The representation $d$ is the representation of $K$ induced from the character $\eta$. Since $\mathscr{O}_{d}$ contains at least one element of $\widehat{L}_{-}$,

$$
\sum_{\psi \in \mathscr{\theta}_{d}} \int_{L_{+}} \psi\left(X_{+}\right) d X_{+} \neq 0
$$

Averaging Howe's character formula over $L_{+}$, we see that $\int_{L_{+}} \xi_{d}\left(\exp X_{+}\right) d X_{+} \neq$ 0 , which is equivalent to the statement that $d$ is spherical.

If $\chi$ is replaced by $\operatorname{Ad}^{*}\left(k_{+}\right) \chi$, for $k_{+} \in K_{+}$, then Theorem 2 implies that we obtain the same representation $d \in \widehat{K}$. Therefore, we have associated to each $K_{+}$-orbit in $\widehat{L}_{-}$a representation $d \in\left(K / K_{+} \hat{)}\right.$. Lemma 2 implies that if $d$ is any spherical representation of $K$, then $\mathscr{O}_{d} \cap \hat{\mathbb{L}}_{-}$is nonempty. Hence the mapping from the space of $K_{+}$-orbits in $\widehat{L}_{-}$to the space $\left(K / K_{+}\right)$is surjective. Injectivity is equivalent to the assertion that if $d \in\left(K / K_{+}\right)$then $\mathscr{O}_{d} \cap \widehat{L}_{-}$ consists of a single $K_{+}$-orbit. However, for $\chi \in \widehat{L}_{-}$the orbit $\operatorname{Ad}^{*}\left(K_{+}\right) \chi$ has $\#\left(L_{+} / I_{+}\right)$elements, since, according to [5], the stabilizer of $\chi$ is the group $\exp I_{+}$. But \#( $\left.L_{+} / I_{+}\right)$is precisely the dimension of the associated representation $d$. From Lemma 2, we see that this dimension equals the cardinality of $\mathscr{O}_{d} \cap \widehat{L}_{-}$. Hence, injectivity follows.

To complete the proof of Theorem 3, we need to establish the character formula. Toward this end, we note that if $d X_{+}$and $d X_{-}$are Haar measures on $L_{+}$and $L_{-}$, respectively, then

$$
\mu(f)=\int_{L_{+}} \int_{L_{-}} f\left(\exp X_{-} \exp X_{+}\right) d X_{-} d X_{+}
$$


defines a Haar measure on $K$ and

$$
\mu(f)=\int_{L_{+}} \int_{L_{-}} f\left(\exp \left(X_{-}+X_{+}\right)\right) d X_{-} d X_{+} .
$$

(See [2].) It follows that

$$
\int_{L_{+}} \xi_{d}\left(\exp \left(X_{-}+X_{+}\right)\right) d X_{+}=\int_{L_{+}} \xi_{d}\left(\exp X_{-} \exp X_{+}\right) d X_{+}=\xi_{d}^{+}\left(\exp X_{-}\right) .
$$

On the other hand, Howe's character formula when averaged over $X_{-}+L_{+}$ yields

$$
\int_{L_{+}} \xi_{d}\left(\exp \left(X_{-}+X_{+}\right)\right) d X_{+}=\sum_{\chi \in \mathscr{O}_{d} \cap \widehat{L}_{-}} \chi\left(X_{-}\right) .
$$

The character formula of Theorem 3 now follows, which completes the proof of Theorem 3.

Theorem 4 is now an easy consequence of Theorem 3. By Howe's correspondence, elements of $\widehat{K}_{+}$correspond to $K_{+}$-orbits in $\widehat{L}_{+}$. The choice of $l$ identifies each character $\chi$ of $L_{+}$with a character $\chi_{l}$ of $L_{-}$such that $\chi_{l}\left(\imath X_{+}\right)=\chi\left(X_{+}\right)$. This gives a $K_{+}$-equivariant identification of $\hat{L}_{+}$with $\hat{L}_{-}$. Combining this with Theorem 3 , we have an identification of $\widehat{K}_{+}$with $\left(K / K_{+}\right)$ such that if $d_{+} \in \widehat{K}_{+}$corresponds to $d \in\left(K / K_{+}\right) \widehat{\text {, then }}$

$$
\operatorname{dim}\left(d_{+}\right) \xi_{d_{+}}\left(\exp X_{+}\right)=\operatorname{dim}(d) \xi_{d}^{+}\left(\exp \imath X_{+}\right)
$$

for all $X_{+} \in L_{+}$. Evaluating at $X_{+}=0$ gives $\operatorname{dim} d=\left(\operatorname{dim} d_{+}\right)^{2}$. This identity can also be seen from the constructions of $d$ and $d_{+}$as induced representations. Indeed, $\operatorname{dim} d=\#\left(L_{+} / I_{+}\right)=\left(\operatorname{dim} d_{+}\right)^{2}$.

\section{REDUCTIVE GROUPS}

When $\mathfrak{g}$ is reductive, we may state our results in a different form which is more convenient for the applications in [2]. Fix a nontrivial character $\psi_{F}$ of $F$ and a nondegenerate $\operatorname{Ad}(G)$-invariant, symmetric $F$-bilinear form $B$ on $\mathfrak{g}$. Replacing $B(X, Y)$ by $B(X, Y)+B\left(X^{\sigma}, Y^{\sigma}\right)$, we may assume $B\left(X^{\sigma}, Y^{\sigma}\right)=$ $B(X, Y)$ for all $X, Y \in \mathfrak{g}$. If $\mathscr{A}$ is a closed additive subgroup of $\mathfrak{g}$, then $\mathscr{A}^{*}$ denotes the dual subgroup consisting of those $X \in \mathfrak{g}$ such that $\psi_{F}(B(X, Y))$ $=1$ for all $Y \in \mathscr{A}$. Pontryagin duality theory says that $\mathscr{A}^{* *}=\mathscr{A}$ and $\left(\mathscr{A}_{1} \cap \mathscr{A}_{2}\right)^{*}=\mathscr{A}_{1}^{*}+\mathscr{A}_{2}^{*}$ for closed subgroups $\mathscr{A}, \mathscr{A}_{1}$, and $\mathscr{A}_{2}$ of $\mathfrak{g}$. It follows from the $\sigma$-invariance of $B$ that $\mathfrak{g}_{+}^{*}=\mathfrak{g}_{-}$.

If $Z \in \mathfrak{g}$ then there is an associated character $\chi \in \widehat{L}$ defined by $\chi(Y)=$ $\psi_{F}(B(Y, Z))$. In this way, we obtain an identification of $\mathfrak{g} / L^{*}$ with $\widehat{L}$. Similarly, we have an identification of $\left(L / L_{+}\right) \hat{)}=\widehat{L}_{-}$with $\left(L \cap \mathfrak{g}_{+}\right)^{*} / L^{*}=$ $\left(L^{*}+\mathfrak{g}_{-}\right) / L^{*}=\mathfrak{g}_{-} /\left(L^{*} \cap \mathfrak{g}_{-}\right)$. The actions of $K$ and $K_{+}$are transferred to the usual adjoint actions on the Lie algebra.

If $d$ is an irreducible spherical representation of $K$, then the corresponding $K_{+}$-orbit in $\widehat{L}_{-}$is identified with an $\operatorname{Ad}\left(K_{+}\right)$-invariant, $\left(L^{*} \cap \mathfrak{g}_{-}\right)$-invariant subset of $g_{-}$which we denote $\mathscr{O}_{d}^{-}$. The character formula of Theorem 3 becomes

$$
\xi_{d}^{+}\left(\exp X_{-}\right)=\operatorname{dim}(d)^{-1} \sum_{Z \in \mathscr{O}_{d}^{-} /\left(L^{*} \cap_{\left.\mathfrak{g}_{-}\right)}\right.} \psi_{F}\left(B\left(Z, X_{-}\right)\right)
$$


For the example considered in Theorem 4, we have an identification of $\widehat{L}_{+}$ with $\mathfrak{g}_{+} /\left(l^{-1} L^{*} \cap \mathfrak{g}_{+}\right)$such that $Z \in \mathfrak{g}_{+}$determines the character $\chi(X)=$ $\psi_{F}(B(\imath Z, \imath X))$ of $L_{+}$. Each irreducible representation $d_{+}$of $K_{+}$is identified with an $\operatorname{Ad}\left(K_{+}\right)$-invariant, $\left(l^{-1} L^{*} \cap \mathfrak{g}_{+}\right)$-invariant subset of $\mathfrak{g}_{+}$which we denote $\mathscr{O}_{d_{+}}^{+}$. If $d_{+}$and $d$ correspond as in Theorem 4 , then $i \mathscr{O}_{d_{+}}^{+}=\mathscr{O}_{d}^{-}$.

\section{ACKNOWLEGMENT}

We gratefully acknowledge the helpful advice of Professors Howe and Jacquet. We also thank the referee for comments that resulted in a more general statement of Theorem 3 and substantial simplifications in the proof.

\section{BIBLIOGRAPHY}

1. B. Gross, Some applications of Gelfand pairs to number theory, Bull. Amer. Math. Soc. (N.S.) 24 (1991), 277-301.

2. J. Hakim, Admissible distributions on p-adic symmetric spaces, preprint.

3. _ Character relations for distinguished representations, Amer. J. Math. (to appear).

4. Harish-Chandra, Admissible invariant distributions on reductive p-adic groups, Queen's $\mathrm{Pa}-$ pers in Pure and Appl. Math., vol. 48, Queen's Univ., Kingston, ON, 1978, pp. 281-347.

5. R. Howe, Kirillov theory for compact p-adic groups, Pacific J. Math. 73 (1977), 365-381.

6. H. Jacquet and K. F. Lai, A relative trace formula, Compositio. Math. 54 (1985), 243-310.

Department of Mathematics \& Statistics, The American University, Washington, D.C. 20016

E-mail address: JHAKIMQAMERICAN.EDU 